\title{
Listado florístico del Parque Nacional Palenque, CHIAPAS, MÉXICO
}

\author{
Héctor Gómez-Domínguez ${ }^{1}$, Miguel Ángel Pérez Farrera, Josefa Anahí Espinoza Jiménez y \\ Mirna IVETt MARQuez ReYNOSO \\ Herbario Eizi Matuda, Instituto de Biología, Universidad de Ciencias y Artes de Chiapas. \\ Tuxtla Gutiérrez, Chiapas. Libramiento Norte Poniente s/n. Colonia Lajas Maciel. \\ 'Autor para la correspondencia: hectorgomezdominguez@gmail.com
}

\begin{abstract}
Resumen: Se realizó el inventario florístico del Parque Nacional Palenque, Chiapas, México. Se realizaron colectas botánicas durante el año 2008 y revisaron bases de datos de herbario. Se registraron 484 especies, 319 géneros y 100 familias. Las familias más representativas corresponde a Fabaceae (31 géneros y 38 especies) y Rubiaceae (18 géneros y 29 especies). Los géneros más diversos fueron Psychotria con diez especies, Ficus y Piper con ocho especies. Se registraron cuatro especies endémicas a Chiapas, 12 especies incluidas en alguna categoría de riesgo en la NOM- ECOL.-059- 2010 y nueve especies registradas en la Lista Roja de la Unión Internacional para la Conservación de la Naturaleza. La riqueza florística registrada, y los elementos de distribución restringida y bajo estatus de conservación, hacen evidente la importancia de la conservación del Parque Nacional Palenque.

Palabras clave: listado florístico, parque nacional, selva alta perennifolia.
\end{abstract}

\begin{abstract}
A floristic inventory was conducted in the Palenque National Park, Chiapas, México. Botanical collections were made in 2008 and herbarium databases were revised. About 484 species, 319 genera and 100 families were recorded. The most representative families correspond to Fabaceae (31 genera and 38 species) and Rubiaceae (18 genera and 29 species). The most diverse genera were Psychotria with ten species, Ficus and Piper with eight species respectively. Four endemic species for Chiapas were recorded, 12 species were recorded under some risk category in the NOM- ECOL-059-2010 and nine species were recorded in the Red List of the International Union for Conservation of Nature. The floristic richness recorded, restricted floristic element and under conservation status, to make clear the importance of conservation of National Park Palenque.
\end{abstract}

Key words: floristic list, national park, tropical rain forest.

$\mathbf{E}$ Chiapas la selva alta perennifolia (Miranda y Hernández X, 1963) también conocida como bosque tropical perennifolio (Rzedowski, 1978), bosque lluvioso de montaña baja (Lower Montane Rain Forest) o bosque lluvioso de montaña (Montane rain forest, en parte) (Breedlove, 1981), se encuentra distribuido en las partes bajas de la regiones montañosas, en una variedad de geoformas, desde lomeríos suaves con suelos profundos y bien drenados hasta pendientes muy pronunciadas con suelos pedregosos, originalmente este tipo de vegetación ocupaba la mayor parte de las Montañas del Oriente (Selva Lacandona), las partes bajas de las Montañas del Norte y una estrecha franja en la vertiente del Pacífico de la Sierra Madre de Chiapas (González-Espinosa y Ramírez-Marcial, 2013). La mayor parte de este tipo de vegetación ha sido alterada para dedicarla a usos agrope-

cuarios, manteniéndose solamente fragmentos maduros en cañadas rocosas o cimas muy inaccesibles (Levy-Tacher $e t$ al 2002; González-Espinosa et al., 2007; Martínez-Ramos y García-Orth, 2007). Esta pérdida y fragmentación de hábitat modifica la estructura espacial, así como la composición y estructura de la vegetación, afectando la distribución de las especies al crearse paisajes altamente alterados (Arroyo-Rodríguez, 2007; Arroyo et al., 2007) Debido a los diversos problemas ambientales y a la necesidad de conservación de las selvas y su biodiversidad se ha creado un Sistema de Áreas Naturales Protegidas cuya principal intención es conservar zonas y elementos representativos de los diversos ecosistemas presentes en el país, los cuales se caracterizan por una considerable riqueza de flora o fauna, o por la presencia de poblaciones, especies o hábitat que se encuentran 
en alguna categoría de riesgo y requieren un control más estricto por la importancia de su preservación (INE, 2000).

En el estado de Chiapas se han decretado 43 áreas naturales protegidas, que cubren una superficie de 1,285,374.05 ha (equivalente al $17.87 \%$ de la superficie del Estado) del total de áreas naturales protegidas, 25 se encuentran bajo jurisdicción Estatal con 167,413.05 ha y 18 bajo jurisdicción de la Federación con 1,117,961 ha (CONABIO, 2014). Dentro de las reservas Federales se incluye al Parque Nacional de la Zona Arqueológica de Palenque, con una extensión de 1,771 ha, decretado el 11 de julio de 1981 (SPP, 1991; Vargas-Márquez 1997; Vargas-Márquez et al., 2000), localizándose en el límite norte de la Selva Lacandona. Esta y muchas otras Áreas Naturales Protegidas de México tienen como problemática la falta de conocimiento de flora, fauna y microorganismos, por lo cual es necesario una evaluación completa de la diversidad biológica con la que cuenta el país (Gómez-Pompa y Dirzo, 1995). Entre los trabajos que se han desarrollado en el Parque Nacional Palenque se encuentran autores como López-Mendoza (1980), Sol-Sánchez (1993) y Díaz-Gallegos (1996), quienes se enfocaron a estudiar la estructura, composición y el uso de la flora del parque; sin embargo, aunque estos trabajos ayudan en gran medida a solventar el vacío en cuanto a información florística, aún son insuficientes, por lo cual este trabajo tuvo como objetivo obtener un listado florístico preliminar para el Parque Nacional Palenque, conjuntando la información obtenida de colectas, así como de bases de datos de herbario.

\section{Materiales y método}

Zona de estudio. El Parque Nacional Palenque está ubicado entre las coordenadas geográficas, de latitud norte $17^{\circ} 27^{\prime}$ 52 " y $17^{\circ} 30^{\prime} 10^{\prime \prime}$ y de longitud oeste $92^{\circ} 01$ ' $48^{\prime \prime}$ y $92^{\circ}$ 01' 48" (Vargas et al., 2000), se localiza a $8 \mathrm{~km}$ del municipio de Palenque, Chiapas y colinda con los ejidos Las Ruinas, Venustiano Carranza, Adolfo López Mateos y Santa Isabel (SARH; 1993). El relieve que se encuentra en la zona es principalmente montañoso con algunas depresiones en el centro, las pendientes son muy abruptas hasta más del $50 \%$. Sin embargo, se pueden encontrar partes semiplanas con pendientes del $2 \%$ en los alrededores de los templos, el pico más alto es el Cerro de Don Juan a una altura de 800 m s.n.m. (Hardy, 1985). El suelo está constituido geológicamente por terrenos del cretácico medio y superior de la era Mesozoica (Mülleried, 1957). La hidrología del parque es una microcuenca del río Michol, subcuenca del río Chacamax y a su vez forma parte de la región hidrológica del Grijalva-Usumacinta (SARH, 1993). Para la zona de Palenque el clima predominante es cálido-húmedo Am W(e)g con lluvias en verano, con una temperatura media anual de $26{ }^{\circ} \mathrm{C}$ y una precipitación pluvial de $2156 \mathrm{~mm}$ al año siendo el mes más seco mayo con una temperatura de $29.7^{\circ} \mathrm{C}$, el mes más frio enero con $22.4{ }^{\circ} \mathrm{C}$ (García, 1981). Díaz-Gallegos
(1996) menciona que la mayor superficie del Parque Nacional Palenque (43.4\%) equivalente a 769.4 ha corresponde a pastizales inducidos, en segundo término se encuentra la selva alta perennifolia, con el $33.7 \%$ que es igual a 597 ha de la superficie total, le continua en orden de importancia la vegetación secundaria y acahuales en diferentes etapas de regeneración con una superficie total de 387.3 ha equivalente al $21.8 \%$ y el resto de la superficie es ocupado por áreas de recreación en los alrededores de los templos.

Métodos. Se realizaron colectas botánicas durante el año 2008 dentro del polígono del Parque Nacional Palenque. Los puntos de colectas fueron los principales senderos y áreas conservadas del parque. Los ejemplares fueron colectados y procesados de acuerdo al procedimiento Lot y Chiang (1986), el material de herbario recabado fue depositado en la colección del Herbario Eizi Matuda (HEM) del Instituto de Ciencias Biológicas de la Universidad de Ciencias y Artes de Chiapas. Los ejemplares se identificaron a especie a través de bibliografía especializada. Adicionalmente, se revisaron las bases de datos en internet y ejemplares de herbario del CAS, CHIP, DS, ENCB, HEM, IEB, IPN, IE-XAL MO, MEXU, LL-TEX Y UAMIZ; así como los listados elaborados por Díaz-Gallegos (1996) e INE (1984). Para conocer las especies en riesgo se consultó la Norma oficial Mexicana NOM-059 (SEMARNAT, 2010) y la Lista Roja de Especies Amenazadas de la Unión Internacional para Conservación de la Naturaleza (IUCN, 2010), Toda la información de las colectas realizadas y consultadas se integraron en una base de datos de Microsoft Excel 2011. Para organizar el listado se uso para Angiospermas la clasificación de la APG III (Chase y Reveal, 2009) y para los helechos se uso la clasificación de Christenhusz et al. (2011).

\section{Resultados}

Se obtuvo un listado florístico que contiene 484 especies pertenecientes a 318 géneros y 103 familias (Tabla 1) El grupo mejor representado fueron las Magnoliophyta con el $468(96.6 \%)$ de especies. Las familias más diversas fueron Fabaceae 31 géneros y 38 especies y Rubiaceae con 18 gé-

Tabla 1. Datos generales de la flora vascular del Parque Nacional Palenque

\begin{tabular}{lccc}
\hline División & Familias & Géneros & Especies \\
\hline Lycophyta & 1 & 1 & 2 \\
Monilophyta & 9 & 9 & 13 \\
Gimnospermae & 1 & 1 & 1 \\
Eucotiledóneas & 68 & 239 & 345 \\
Magnolidas & 7 & 13 & 32 \\
Monocotiledóneas & 17 & 55 & 91 \\
Total general & 103 & 318 & 484 \\
\hline
\end{tabular}




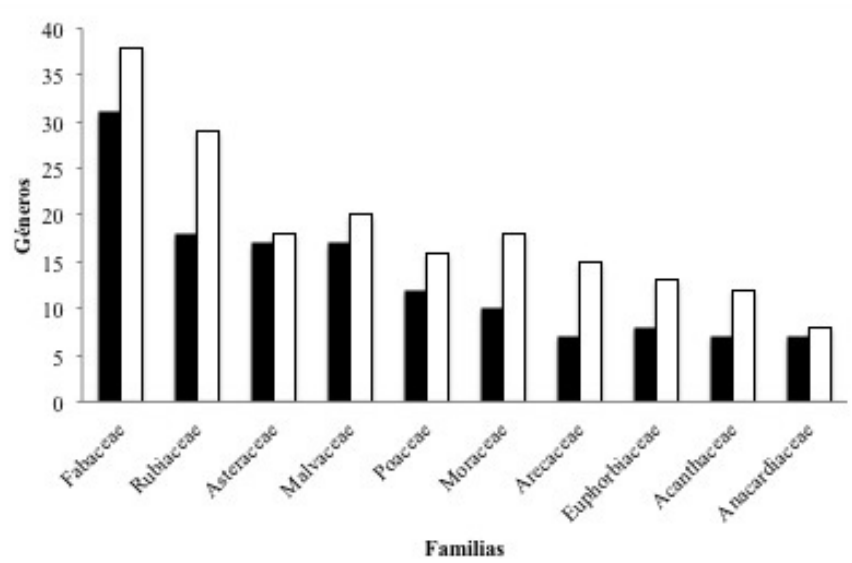

Figura 1. Relación de familias con mayor número de géneros (barras negras) y especies (barras blancas) del Parque Nacional Palenque

neros y 29 especies (Figura 1). Los géneros más diversos fueron Psychotria con diez especies, Ficus y Piper con ocho especies cada una (Figura 2). De acuerdo a la forma de crecimiento, los árboles y las hierbas fueron las más abundantes con 210 y 183 especies respectivamente. De las especies de flora endémica se encontraron, cinco especies endémicas de Chiapas, dos especies de distribución restringida (Chiapas-Oaxaca y Chiapas-Guatemala) y 142 de México a Centroamérica (Apéndice 1)

Se registraron 12 especies incluidas en alguna categoría de riesgo en la NOM- ECOL.-059- 2010 y nueve especies se encuentran en la Lista Roja de la Unión Internacional para la Conservación de la Naturaleza (Tabla 2) de acuerdo a esta

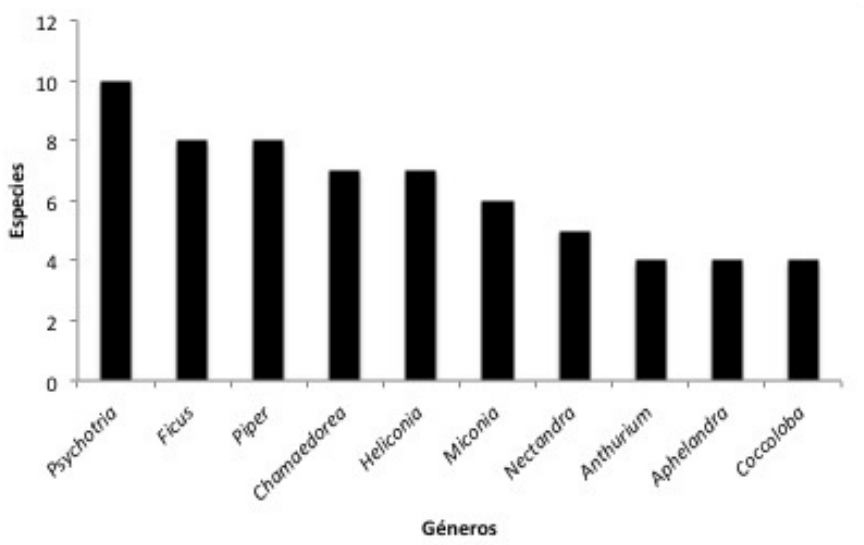

Figura 2. Relación de géneros con mayor número de especies en el Parque Nacional Palenque.

lista, Nectandra leucocome, Trichilia breviflora y Zamia lacandona son especies en peligro de extinción.

\section{Discusión}

La comparación con otros trabajos resulta difícil debido a los objetivos y a los diferentes métodos utilizados. El Parque Nacional Palenque alberga una riqueza de flora que equivale al $6.1 \%$ de la flora registrada para el Estado de Chiapas (Breedlove, 1986) y representa el $16.7 \%$ de la flora registrada para La Selva Lacandona, una de las mayores áreas con bosque tropical perennifolio del Estado de Chiapas (Martínez et al., 1994), así mismo las magnoliophytas registradas

Tabla 2. Especies del Parque Nacional Palenque que se encuentran bajo alguna categoría de riesgo en la NOM-ECOL-059-2010 (A = Amenazada, P = Peligro de extinción, Pr = Protección) y en la lista roja de especies en peligro de la IUCN. 2010 (EN = Peligro de extinción, LR/NT = Riesgo menor, $\mathrm{VU}=$ Vulnerable).

\begin{tabular}{lccc}
\hline Especie & Familia & Estatus & Distribución \\
\hline Acosmium panamens & Fabaceae & NOM A & No endémica \\
Andira galeottiana & Fabaceae & IUCN VU & No endémica \\
Aspidosperma megalocarpon & Apocynaceae & No endémica \\
Astronium graveolens & Anacardiaceae & No endémica \\
Calophyllum brasiliense & Clusiaceae & NOM A & No endémica \\
Cedrela odorata & Meliaceae & NOM A & No endémica \\
Chamaedorea arenbergiana & Arecaceae & No endémica \\
Chamaedorea cataractarum & Arecaceae & Endémica \\
Guatteria anomala & Annonaceae & NOM A & No endémica \\
Huperzia dichotoma & Lycopodiaceae & No endémica \\
Monstera tuberculata & Araceae & No endémica \\
Nectandra leucocome & Lauraceae & NOM A; IUCN LR/NT & Endémica \\
Saurauia leucocarpa & Actinidaceae & NOM A & NOM EN \\
Spathiphyllum friedrichsthalii & Araceae & IUCN VU & NOM A \\
Swietenia macrophylla & Meliaceae & IUCN VU \\
Trichilia breviflora & Melicaceae & IUCN EN & NOM P \\
Vatairea lundellii & Fabaceae & Zamiaceae & NOM P; IUCN EN \\
Zamia lacandona & & No endémica \\
\hline
\end{tabular}


Tabla 3. Comparación de la riqueza florística del Parque Nacional Palenque con otras áreas naturales protegidas o regiones. * Los trabajos de Meave (1983) y Duran-Fernández (1999) abarcan sólo una hectárea del total del área natural protegida

\begin{tabular}{|c|c|c|c|}
\hline $\begin{array}{l}\text { Área Natural } \\
\text { Protegida }\end{array}$ & $\begin{array}{l}\text { Superficie } \\
\mathrm{Ha}\end{array}$ & $\begin{array}{l}\text { Número } \\
\text { especies }\end{array}$ & Fuente \\
\hline Chiapas & $7,441,500$ & 8,248 & Breedlove, 1986 \\
\hline $\begin{array}{l}\text { Montes Azules } \\
\text { (Selva Lacandona) }\end{array}$ & $1,550,200$ & 3,400 & Martínez et al., 1994 \\
\hline $\begin{array}{l}\text { Reserva de la } \\
\text { Biosfera La Sepultura }\end{array}$ & 167,309 & 1,798 & Reyes-García, 2008 \\
\hline Los Tuxtlas, Veracruz & 155,000 & 3,000 & Ruíz y Duran, 2004 \\
\hline $\begin{array}{l}\text { Reserva de la } \\
\text { Biosfera El Triunfo }\end{array}$ & 119,117 & 3,000 & Pérez-Farrera, 2004 \\
\hline $\begin{array}{l}\text { Reserva de la } \\
\text { Biosfera El Ocote }\end{array}$ & 48,000 & 705 & Ochoa-Gaona, 1996 \\
\hline $\begin{array}{l}\text { Parque Nacional } \\
\text { Cañón del Sumidero }\end{array}$ & 21,789 & 1,298 & $\begin{array}{l}\text { Espinoza-Jiménez } \\
\text { et al., } 2011\end{array}$ \\
\hline $\begin{array}{l}\text { Monumento } \\
\text { Natural Yaxchilán }\end{array}$ & 2,261 & 547 & Meave et al., 2008 \\
\hline $\begin{array}{l}\text { Parque Nacional } \\
\text { Palenque }\end{array}$ & 1,771 ha & 484 & Este trabajo \\
\hline $\begin{array}{l}\text { Parque Educativo } \\
\text { Laguna Bélgica }\end{array}$ & 47.5 ha & 254 & $\begin{array}{l}\text { Escobar-Ocampo y } \\
\text { Ochoa-Gaona, } 2007\end{array}$ \\
\hline $\begin{array}{l}\text { Monumento } \\
\text { Natural Bonampak }\end{array}$ & 1 ha & 267 & Meave, 1983 \\
\hline $\begin{array}{l}\text { Área de Protección } \\
\text { de Flora y Fauna Nahá }\end{array}$ & 1 ha & 283 & $\begin{array}{l}\text { Duran-Fernández, } \\
1999\end{array}$ \\
\hline
\end{tabular}

en este trabajo representan el $2.14 \%$ de las especies reportadas para el país y el $5.9 \%$ de las reportadas para Chiapas (Villaseñor y Ortiz, 2014). La riqueza florística registrada en el Parque Nacional Palenque es similar a la reportada por Meave et al. (2008), aun cuando la superficie de Yaxchilán es el doble que el Parque Nacional Palenque, la diferencia observada con otras áreas o reservas se debe principalmente que éstas abarcan diferentes tipos de vegetación mientras que Palenque solamente engloba un tipo, la selva alta perennifolia (Tabla 3) Fabaceae y Rubiaceae son las familias más diversas dentro del Parque Nacional Palenque, la primera de ellas concuerda con lo encontrado en el Monumento Natural Yaxchilán por Meave et al. (2008) y en la región de Lancaha-Chansajab por Levy-Tacher et al (2006); y ambas se encuentran entre las quince con mayor número de especies dentro del país (Villaseñor, 2003). Los géneros con mayor número de especies son Psychotria, Ficus y Piper; el primero y el tercero de estos géneros concuerda con el trabajos realizados en el área de protección de flora y fauna Naha (Durán-Fernández, 1999) y Yaxchilán (Meave et al., 2008), ambos géneros son reconocidos por su elevado número de especies dentro del país (Villaseñor, 2004). El género Ficus es uno de los más representativos del bosque tropical perennifolio, pudiendo muchas veces formar bosques (Rzedows- ki, 1978). La zona que cubre el Parque Nacional Palenque protege 12 especies dentro de la NOM- ECOL.-059- 2010 y al bosque tropical perennifolio, el cual ha tenido una disminución dramática de su extensión debido a la colonización de áreas para tierras de cultivo y ganadería (March y Flamenco, 1996), además de especies en peligro de extinción y endémicas como son Zamia lacandona (Stevenson, 2010) y Chamaedorea cataractarum (Hodel, 1992). Se registra por primera vez para la flora de Chiapas la existencia de Ocotea magnifolia (Lundell) Lundell. Esta especie solamente había sido registrada para los estados de Oaxaca, Tabasco y Veracruz. Tomando las dimensiones de este Parque, la riqueza florística es elevada, con elementos de distribución restringida y bajo estatus de conservación; pero sobre todo el bosque tropical perennifolio que contiene, hace evidente la necesidad de fomentar planes de restauración de áreas degradadas.

\section{Agradecimientos}

El primer autor agradece a la dirección y al personal del Parque Nacional Palenque. Al Dr. Christopher Davidson y Sharon Christoph por el apoyo dentro del proyecto "Diversidad florística y ecología evolutiva en áreas naturales protegidas de Chiapas".

\section{Literatura citada}

Arroyo-Rodriguez V. 2007. Calidad de hábitat y distribución de monos aulladores en tres paisajes fragmentados de los Tuxtlas, México. Tesis de Doctorado.Instituto de Ecología A. C. Xalapa, Veracruz, México. 118 pp.

Arroyo-Rodríguez V., Mandujano S., Benítez-Malvido J. y Cuende-Fanton C. 2007. The influence of large tree density of Howler Monkey (Alouatta palliata mexicana) presence in very small rain forest fragments. Biotropica 39:760-766

Breedlove D.E. 1981. Flora of Chiapas. Part I. Introduction to the Flora of Chiapas. California Academy of Sciences, San Francisco.

Breedlove D.E. 1986. Listados Florísticos de México VI. Flora de Chiapas. Instituto de Biología. Universidad Nacional Autónoma de México. México, D.F.

Chase M.W. y Reveal J.L. 2009. A phylogenetic classification of the land plants to accompany APG III. Botanical Journal of the Linnean Society. 161:122-127.

Christenhusz M., Zhang X-H. y Schneider H. 2011. A linear sequence of extant families and genera of lycophytes and ferns. Phytotaxa 19: 7-54.

CONABIO [Comisión Nacional para el Conocimiento y Uso de la Biodiversidad]. 2014. Sitios prioritarios de conservación en Chiapas shttp:// www. biodiversidad. gob.mx /corridor /cbmm /DOC/41_308.pdf> (consultado el 8 de Julio de 2014).

Díaz-Gallegos J.R. 1996. Estructura y vegetation florística del Parque Nacional Zona Arqueológica de Palenque, Chiapas. Tesis de Licenciatura, División Académica de Ciencias Biológicas, Universidad Juárez Autónoma de Tabasco. Villahermosa, México 63 p. 
Durán-Fernández A. 1999. Estructura y etnobotánica de la selva alta perennifolia de Naha, Chiapas. Tesis de Maestría, Facultad de Ciencias, Universidad Autónoma de México, México D.F. $150 \mathrm{pp}$.

Escobar-Ocampo M.C. y Ochoa-Gaona S.,2007. Estructura y composición florística de la vegetación del Parque Educativo Laguna Bélgica, Chiapas, México. Revista Mexicana de Biodiversidad. 78:391-419

Espinosa-Jiménez J.A., Pérez Farrera M.A. y Martínez Camilo R. 2011. Inventario florístico del Parque Nacional Cañón del Sumidero, Chiapas, México. Boletín de la Sociedad Botánica de México. 89: 37-82.

García E. 1981. Modificaciones al sistema de clasificación climática de Köppen para adaptarlo a las condiciones de la República Mexicana. Offset Larios S.A., México, D.F.

Gómez-Pompa A. y Dirzo R. (Coords) 1995. Reservas de la biosfera y otras áreas naturales protegidas de México. Instituto Nacional de Ecología,

González-Espinosa M. y Ramírez-Marcial N. 2013. Comunidades vegetales terrestres. En: Comisión Nacional para el Conocimiento y uso de la Biodiversidad (CONABIO). La Biodiversidad en Chiapas. Estudio de Estado, pp. 21-42. Comisión Nacional para el Conocimiento y uso de la Biodiversidad / Gobierno del Estado de Chiapas, México.

González-Espinosa M., Ramírez-Marcial N., Camacho-Cruz A., Holz S.C., ReyBernays.J.M. y Parra-Vázquez M.R. 2007. Restauración de bosques en territorios indígenas de Chiapas: Modelos ecológicos y estrategias de acción. Boletín de la Sociedad Botánica de México. 80:11-23.

Hodel, D.R. 1992. Chamaedorea palms. The species and their cultivation. Allen, Kansas. 338 p.

Instituto Nacional de Ecología. 1984. Propuesta de inscripción del Parque Nacional Palenque al patrimonio mundial, cultural y natural de la UNESCO, SEDUE.

$<$ http://repositorio.inecc.gob.mx/ae/ae_002271.pdf>, (descargado el 7 de enero del 2014).

Instituto Nacional de Ecología. 2000. Estrategia Nacional para la Vida Silvestre. Logros y Retos para el Desarrollo Sustentable 195-2000. Secretaría de Medio Ambiente, Recursos Naturales y Pesca. México.

Hardy A. 1985. Palenque pasado y presente. Monografía. Gobierno del Estado de Chiapas. Tuxtla Gutiérrez, 96 pp.

IUCN [International Union for Conservation of Nature]. 2010. IUCN Red List of Threatened Species. Version 2010. <http:// www.iucnredlist.org/>. (consultado 1 a 12 de diciembre de 2013).

Levy-Tacher S.I., Aguirre-Rivera J.R., Martínez-Romero M.M. y Durán-Fernández A. 2002. Caracterización del uso tradicional de la flora espontánea en la comunidad lacandona de Lacanhá, Chiapas, México. Interciencia 27:512-520.

Levy-Tacher S.I., Aguirre-Rivera J.R., García-Pérez J.D. y Martínez-Romero M.M. 2006. Aspectos florísticos de Lacanhá Chansayab, Selva Lacandona, Chiapas. Acta Botanica Mexicana 77:69-98.

López-Mendoza R. 1980. Tipos de vegetación y su distribución en el Estado de Tabasco y norte de Chiapas. Universidad Autónoma de Chapingo. Cuadernos Universitarios, Agronomía 1. México D. F. 121 pp.

Lot A. y Chiang F. 1986. Manual de Herbario: Administración y Manejo de Colecciones, Técnicas de Recolección y Prepara- ción de Ejemplares Botánicos. Consejo Nacional de la Flora de México. México, D.F.

Martínez E., Ramos A.C. y Chiang F. 1994. Lista florística de la Lacandona, Chiapas. Boletín de la Sociedad Botánica de México 54:99-117.

Martínez-Ramos M. y García-Orth X. 2007. Sucesión ecológica y restauración de las selvas húmedas. Boletín de la Sociedad Botánica de México 80:69-84.

Meave J.A. 1983. Estructura y composición de la selva alta perennifolia en los alrededores de Bonampak, Chiapas. Tesis de Licenciatura, Facultad de Ciencias, Universidad Autónoma de Chiapas, México D. F. 140 pp.

Meave J.A., Romero-Romero M.A., Valle-Doménech A., RincónGutiérrez A., Martínez E. y Ramos C.H. 2008. Plant diversity assessment in the Yaxchilán Natural Monument, Chiapas, México. Boletín de la Sociedad Botánica de México 83:53-76.

March-Mifsut I.J. y Flamenco-Sandoval A. 1996. Evaluación rapida de la deforestación en la áreas naturales protegidas de Chiapas (1970-1993). El Colegio de la Frontera Sur. San Critóbal de las Casas.

Miranda F. y Hernández X. E. 1963. Los tipos de vegetación de México y su clasificación. Boletín de la Sociedad Botánica de México 29:1-179.

Mülleried F.K.G. 1957. Geología de Chiapas. Gobierno del Estado de Chiapas, Tuxtla Gutiérrez.

Ochoa-Gaona S. 1996. La vegetación de la Reserva El Ocote a lo largo del Cañón del río LA Venta. En: Vásquez-Sánchez M.A, March- Mifsut I. Eds. Conservación y Desarrollo Sustentable en la Selva El Ocote, Chiapas, pp. 45-86, El Colegio de la Frontera Sur-Comisión Nacional para el Conocimiento y Uso de los Recursos Naturales, A. C., San Cristóbal de las Casas, México.

Pérez-Farrera M.A. 2004. Flora y vegetación de la Reserva de la Biosfera El Triunfo: Diversidad, riqueza y endemismos. En: Pérez-Farrera M.A., Martínez-Meléndez N., Hernández-Yánez A. y Arreola-Muñoz A.V. Eds. La Reserva de la Biosfera El Triunfo, Tras Una Década de Conservación, pp. 77-100, Universidad de Ciencias y Artes de Chiapas, México, D. F.

Reyes-García A.J. 2008. Inventario florístico de la reserva de la biosfera La Sepultura, Sierra Madre de Chiapas. Tesis de Maestría, Facultad de Ciencias, Universidad Autónoma de México, México, D.F. 195 pp.

Rzedowski J. 1978. Vegetación de México. Limusa Wiley, México, D.F.

Ruiz C.J. y Durán L. 2004. La Estación Biológica Tropical “Los Tuxtlas” (Veracruz: México) ¿Conviene Una Actitud Preservacionista? Universidad Nacional Autónoma de México. México. D.F.

SEMARNAT [Secretaría del Medio Ambiente y Recursos Naturales]. 2010. Norma Oficial Mexicana NOM-059-SEMARNAT-2010, Protección ambiental - Especies nativas de México de flora y fauna silvestres - Categorías de riesgo y especificaciones para su inclusión, exclusión o cambio - Lista de especies en riesgo. Diario Oficial de la Federación. 2da Sección, 30 de diciembre de 2010.

Stevenson D.W. 2010. Zamia lacandona. En: IUCN (International Union for Conservation of Nature) 2013. IUCN Red List of Threatened Species. Version 2013.2. <www.iucnredlist.org>. (consultado 09 Junio de 2014)

SARH. 1993. Diagnóstico del Parque Nacional Palenque. Subsecretaría Forestal y de Fauna Silvestre. Consultoría Wildlife. 
Sol-Sánchez A. 1993. Utilización de los recursos vegetales por los habitantes del Ejido Linda Vista, Palenque, Chiapas. Tesis profesional, División Académica de Ciencias Biológicas. Universidad Juárez Autónoma de Tabasco, Tabasco, México 86 pp.

Vargas-Márquez F. 1997. Parques Nacionales de México, Aspectos físicos, sociales, legales, administrativos, recreativos, biológicos, culturales, situación actual y propuestas en torno a los Parque Nacionales de México. Instituto Nacional de EcologíaSEMARNAP

Vargas-Marquez F., Escobar S. y Del-Ángel R. (Comp.) 2000. Áreas Naturales Protegidas de México con decretos federales
(1899. 2000). ISBN 968-817-376-2. Instituto Nacional de Ecología - SEMARNAP, Red para el Desarrollo Sostenible, A. C.

Villaseñor J.L. 2003. Diversidad y distribución de las Magnoliophyta en México. Interciencia 28:160-167.

Villaseñor J.L. 2004. Los géneros de plantas vasculares de la flora de México. Boletín de la Sociedad Botánica de México 75:105135.

Villaseñor J.L. y Ortíz E. 2014. Biodiversidad de las plantas con flores (División Magnoliophyta) en México. Revista Mexicana de Biodiversidad 85:134-135.

Recibido: 5 de junio de 2014

Aceptado: 15 de julio de 2014 
Apéndice 1. Lista florística de la vegetación del Parque Nacional Palenque, Chiapas, México. (FC): Á= Árbol, Ar. Arbusto, AB= Arborescente, $\mathrm{H}=$ Hierba, $\mathrm{B}=$ Bejuco o Liana. Distribución: $\mathrm{CA}=$ Centro américa, Cosp. = Cosmopolita, Chis=Chiapas, Gua = Guatemala, Oax= Oaxaca, Car= Caribe, SA= Suramérica, NA= Norteamérica. Nombres de los colectores en apéndice 2

\begin{tabular}{|c|c|c|}
\hline Familia/Especie/ Colector & FC & Distribución \\
\hline $\begin{array}{l}\text { TRACHEOPHYTA } \\
\text { LYCOPHYTA } \\
\text { Lycopodiaceae }\end{array}$ & & \\
\hline Lycopodium dichotomum Jacq. INE & $\mathrm{H}$ & Méx, CA. \\
\hline Lycopodium cernuum L., Matuda 3781 & $\mathrm{H}$ & Méx, CA. \\
\hline \multicolumn{3}{|l|}{ MONILOPHYTA } \\
\hline \multicolumn{3}{|l|}{ Dennstaedtiaceae } \\
\hline Pteridium aquilinum (L.) Huhn INE & $\mathrm{H}$ & Cosp. \\
\hline \multicolumn{3}{|l|}{ Dryopteridaceae } \\
\hline Ctenitis melanosticta (Kuntze) Copel., Díaz-Gallegos 367 & $\mathrm{H}$ & Méx, CA. \\
\hline Thelypteris meniscioides (Liebm.) C.F.Reed, Spellman et al 60 & $\mathrm{H}$ & Méx, Gua. \\
\hline \multicolumn{3}{|l|}{ Lomariopsidaceae } \\
\hline \multicolumn{3}{|l|}{ Lygodiaceae } \\
\hline Lygodium heterodoxum Kunze, Matuda 3716 & $\mathrm{H}$ & Méx, SA. \\
\hline Lygodium venustum SW. Matuda 3720 & $\mathrm{H}$ & Méx, SA. \\
\hline \multicolumn{3}{|l|}{ Polypodiaceae } \\
\hline Campyloneurum angustifolium (Sw.) Fée, Matuda 3705 & $\mathrm{H}$ & Cosp. \\
\hline Adiantum latifolium Lam., Matuda 3678 & $\mathrm{H}$ & Méx, SA. \\
\hline Adiantum pulverulentum L., INE & $\mathrm{H}$ & Méx, SA. \\
\hline \multicolumn{3}{|l|}{ Schizaeaceae } \\
\hline Schizaeae elegans (Vahl) Sm., Matuda 3662 & $\mathrm{H}$ & Méx, SA. \\
\hline \multicolumn{3}{|l|}{ Tectariaceae } \\
\hline Tectaria heracleifolia (Willd.) Underw., SC y GEC 5473, Díaz-Gallegos 369 & $\mathrm{H}$ & NA, Méx, CA, SA. \\
\hline Tectaria incisa Cav. INE & $\mathrm{H}$ & Méx, CA. \\
\hline \multicolumn{3}{|l|}{ Thelypteridaceae } \\
\hline Thelypteris patens (Sw.) Small, EFC \& HC 1945 & $\mathrm{H}$ & Méx, CA. \\
\hline \multicolumn{3}{|l|}{ SPERMATOPHYTA } \\
\hline \multicolumn{3}{|l|}{ GIMNOSPERMAE } \\
\hline \multicolumn{3}{|l|}{ Zamiaceae } \\
\hline Zamia lacandona Schutzman \& Vovides, RMC y NMM s/n & $\mathrm{AB}$ & Chis. \\
\hline \multicolumn{3}{|l|}{ ANGIOSPERMAE } \\
\hline \multicolumn{3}{|l|}{ EUCOTILEDÓNEAS } \\
\hline \multicolumn{3}{|l|}{ Acanthaceae } \\
\hline Aphelandra aurantiaca (Scheidw) Lindl. INE; Díaz-Gallegos 382 & $\mathrm{H}$ & Méx, CA, SA. \\
\hline Aphelandra deppeana Schltdl. \& Cham Díaz-Gallegos 111 & $\mathrm{H}$ & Méx, CA. \\
\hline Aphelandra schiedeana Schltdl. \& Cham Díaz-Gallegos 345 & $\mathrm{H}$ & Méx, CA. \\
\hline Aphelandra speciosa Brandegee INE & $\mathrm{H}$ & Méx, Gua. \\
\hline Blechum pyramidatum (Lam.) Urb. Díaz- Gallegos 288 & $\mathrm{H}$ & NA, Méx, CA, SA. \\
\hline Justicia aurea Schldt. Díaz-Gallegos 277 & $\mathrm{H}$ & Méx, CA. \\
\hline Justicia spicigera (Schltdl.) L. H. Bailey INE & $\mathrm{H}$ & Méx, CA. \\
\hline Louteridium mexicanum (Baill.) Standl INE & $\operatorname{Ar}$ & Méx, Gua. \\
\hline Louteridium donell-smithii S. Watson Díaz-Gallegos 366 & Á & Méx, CA. \\
\hline Mirandea sylvatica Acosta Cast. TLW 1987 & $\mathrm{H}$ & Méx. \\
\hline $\begin{array}{l}\text { Odontonema callistachyum (Schltdl. \& Cham) Kuntze. Matuda 3694; } \\
\text { Díaz-Gallegos 10, 117; FVA } 19988\end{array}$ & $\mathrm{Ar}$ & Méx, CA. \\
\hline Ruellia geminiflora Kunth Díaz-Gallegos s/n & $\mathrm{H}$ & Méx, CA, SA. \\
\hline
\end{tabular}


Apéndice 1. Continuación

Familia/Especie/ Colector

\section{Actinidiaceae}

Saurauia leucocarpa Schltdl. EFC y HC s/n; Matuda 3700

Saurauia rubiformis Vatke RMC y NMM 1223

Saurauia yasicae Loes. EFC y HC 7006A; RMC y NMM 1221; MJH y

EFC 2442; Spellman et al. 169

\section{Amaranthaceae}

Achyranthes aspera L. RFT s/n; EL 40567

Amaranthus hybridus L. INE

Amaranthus spinosus L. INE

Celosia argentea L. Díaz-Gallegos 326

Cyathula achyranthoides (Kunth) Moq OFC 26

Iresine diffusa Humb. \& Bonpl. ex Willd. INE

Iresine nigra Uline \& W.L. Bray. RMC, NMM y JMM 1222

Gomphrena decumbens Jacq. EFC y HC 5308

\section{Anacardiaceae}

Astronium graveolens Jacq. JMM 1935; Pennington y Sarukhán s/n

Mangifera indica L. INE, Díaz-Gallegos 309

Metopium brownei (Jacq.) Urb. INE

Mosquitoxylum jamaicense Krug \& Urb. HGD s/n

Toxicodendron striatum (Ruiz \& Pav.) Kuntze Matuda 3605

Spondias mombin L. INE, Díaz-Gallegos 145, 314

Spondias purpurea L. Díaz-Gallegos 298

Tapirira mexicana Marchand RMC y NMM 1208

Apocynaceae

Aspidosperma megalocarpon Müll. Arg. Díaz-Gallegos 85, 395; DD 314;

JMM 1922; NMM y JMM 1229;

Plumeria rubra L. Pennington y Saruknán s/n

Stemmadenia donnell-smithii (Rose) Woodson EFC et al. s/n;

Díaz-Gallegos 137, 236, 161, 232

Stemmadenia eubracteata Woodson HGD $s / n$

Stemmadenia pubescens Benth Díaz- Gallegos 119; RMC y

NMM 1213, 1220; DD 313

Tabernaemontana alba Mill. JMM 1915, 1932

Thevetia ahouai (L.) A. DC. OFC 2

Urechites andrieuxii Müll. Arg. Matuda 3677

\section{Aquifoliaceae}

Ilex guianensis (Aubl.) Kuntze Matuda 3125

\section{Araliaceae}

Dendropanax arboreus (L.) Decne. \& Planch. RMC y NMM s/n, EFC y

HC s/n; RFT s/n, EL 40538, Díaz-Gallegos 28, 326

Dendropanax populifolius (Marchal) A.C. Sm. JMM 1924

Didymopanax morototoni (Aubl.) Decne. \& Planch. Pennington y Saruknán s/n

Oreopanax capitatus (Jacq.) Decne. \& Planch. INE

Oreopanax obtusifolius L.O. Williams EFC y HC 3124

Oreopanax xalapensis (Kunth) Decne. \& Planch. López-Mendoza s/n

\section{Asclepiadaceae}

Asclepias curassavica L. Díaz- Gallegos 311

Gonolobus barbatus Kunth. Díaz- Gallegos 316

Asteraceae

Ageratum corymbosum Zuccagni. Díaz-Gallegos 12

FC

Distribución

Á

Á

Á

$A$

H

$\mathrm{H}$

$\mathrm{H}$

$\mathrm{H}$

$\mathrm{H}$

$\mathrm{H}$

$\mathrm{H}$

$\mathrm{H}$

Á

Á

Á

Á

Á

Á

Á

Á

Á

Á

Á

Á

Á

Á

Á

B

Á

Á

Á

Á

Ar

Á

Á

$\mathrm{H}$

B

$\mathrm{H}$
Méx, CA.

Méx, CA.

Méx, CA, SA.

Cosp.

Cosp.

Cosp.

Cosp.

Cosp.

Méx, CA, SA.

Méx, CA.

NA, Méx, CA, SA.

Méx, CA, SA.

Cosp.

Méx, CA, Car.

Méx, CA, Car.

Méx, CA.

Méx, CA, SA.

NA, Méx, CA, SA, Car.

Méx, CA.

Méx, CA, SA.

Méx, CA, SA, Car.

Méx, CA.

Méx, CA.

Méx, CA.

NA, Méx, CA, Car.

Méx, CA, SA.

NA, Méx, CA.

Méx, CA, SA, Car.

Méx, CA, SA.

Méx.

Méx, CA, SA.

Méx, CA, SA, Car.

Méx, CA.

Méx, CA.

Cosp.

Méx, CA.

NA, Méx, CA. 
Apéndice 1. Continuación

\begin{tabular}{|c|c|c|}
\hline Familia/Especie/ Colector & FC & Distribución \\
\hline Ageratum microcephalum HemsI. INE & $\mathrm{H}$ & Méx, Gua. \\
\hline Archibaccharis serratifolia (Kunth) S.F. Blake INE & $\mathrm{H}$ & Méx, Gua. \\
\hline Bidens pilosa L. INE & $\mathrm{H}$ & Cosp. \\
\hline Calea trichotoma Donn. Sm. Matuda 3755 & $\mathrm{H}$ & Méx, CA. \\
\hline Conyza bonariensis (L.) Cronquist INE & $\mathrm{H}$ & NA, Méx, CA, SA, Car. \\
\hline Erygeron canadensis L. INE & $\mathrm{H}$ & Cosp. \\
\hline Chaptalia nutans (L.) Pol. Matuda 3818 & $\mathrm{H}$ & NA, Méx, CA, SA, Car. \\
\hline Dahlia coccinea Cav. INE & $\mathrm{H}$ & Méx, CA. \\
\hline Fleischmannia pycnocephala (Less.) R. M. King \& H. Rob INE & $\mathrm{H}$ & NA, Méx, CA, SA, Car. \\
\hline Hebeclinium macrophyllum L. INE & $\mathrm{H}$ & Méx, CA, SA. \\
\hline Melanthera nivea (L.) Small Díaz- Gallegos 291 & $\mathrm{H}$ & NA, Méx, CA, SA, Car. \\
\hline Neurolaena lobata (L.) Cass. INE; WSH 164 & $\mathrm{H}$ & NA, Méx, CA, SA. \\
\hline Pectis bonplandiana Kunth DEB 49832 & $\mathrm{H}$ & Méx, CA, SA. \\
\hline Symphyotrichum subulatum (Michx.) G.L. Nesom. Días- Gallegos 6; Matuda 3631 & $\mathrm{H}$ & Cosp. \\
\hline Tithonia diversifolia (Hemsl.) A.Gray INE & $\mathrm{H}$ & Cosp. \\
\hline Vernonia patens Kunth. INE & $\mathrm{H}$ & Méx, CA, SA. \\
\hline \multicolumn{3}{|l|}{ Begoniaceae } \\
\hline Begonia fischeri Schrank. Díaz-Gallegos 61 & $\mathrm{H}$ & Méx, CA, SA. \\
\hline Begonia heracleifolia Schltdl. \& Cham. EFC et al. 2684; WSH 39 & $\mathrm{H}$ & Méx, CA. \\
\hline Begonia glabra Aubl. INE & $\mathrm{H}$ & Méx, CA, SA, Car. \\
\hline \multicolumn{3}{|l|}{ Bignoniaceae } \\
\hline Amphitecna apiculata A.H. Gentry RMC y NMM 1211 & Á & Méx, Gua. \\
\hline Amphitecna latifolia (Mill.) INE & Á & Méx, CA, SA. \\
\hline Arrabidaea inaequalis (DC. ex Slitg.) Bureau ex K. Schum. Matuda 3668 & B & Méx, CA, SA. \\
\hline Arrabidaea verrucosa (Standl.) A. H. Gentry EFC y HC 5301 & $\mathrm{~B}$ & Méx, CA, SA. \\
\hline Macfadyena unguis -cati (L.) A. H. Gentry INE & B & Cosp. \\
\hline Parmentiera aculeata (Kunth) Seem. Díaz- Gallegos 33, 310 & Á & NA, Méx, CA, SA. \\
\hline Tabebuia chrysantha (Jacq.) G. Nicholson. Pennington y Sarukhán s/n & Á & Méx, CA, SA. \\
\hline Tabebuia rosea (Bertol.) A. DC. RMC y NMM 1932, Díaz-Gallegos 389 & Á & Méx, CA, SA. \\
\hline \multicolumn{3}{|l|}{ Bixaceae } \\
\hline Bixa orellana L. INE & Á & Méx, CA, SA. \\
\hline Cochlospermum vitifolium (Willd.) Spreng. Díaz-Gallegos 7 & Á & Méx, CA, SA, Car. \\
\hline \multicolumn{3}{|l|}{ Boraginaceae } \\
\hline Bourreria mollis Standl. Matuda 3682 & Á & Méx, CA, SA. \\
\hline Cordia alliodora (Ruiz \& Pav.) Cham OFC 8, Pennington y Sarukhán s/n. & Á & Méx, CA, SA. \\
\hline Cordia stenoclada I. M. Johnst. Matuda 3695 & Á & Méx, Gua. \\
\hline Heliotropium angiospermum Murray INE & Á & Méx, CA, SA, Car. \\
\hline Tournefortia bicolor Sw. INE & $\operatorname{Ar}$ & Méx, CA, SA. \\
\hline \multicolumn{3}{|l|}{ Burseraceae } \\
\hline $\begin{array}{l}\text { Bursera simaruba (L.) Sarg. RFT s/n; EL 40525; JMM 1918; RMC y } \\
\text { NMM 1218; Díaz-Gallegos 64, HGD } 1926\end{array}$ & Á & NA, Méx, CA, SA. \\
\hline Protium copal (Schltdl. \& Cham.) Engl. INE & Á & Méx, CA. \\
\hline \multicolumn{3}{|l|}{ Cactaceae } \\
\hline Epiphyllum pumilum Britton \& Rose INE & $\mathrm{H}$ & Méx. \\
\hline Pseudorhipsalis ramulosa (Salm-Dyck) Barthlott RMC, NMM y JMM s/n & $\mathrm{H}$ & Méx, CA, SA, Car. \\
\hline $\begin{array}{l}\text { Rhipsalis baccifera (J.S. Muell.) Stearn JMM 1906; RMC, NMM y } \\
\text { JMM s/n; Díaz-Gallegos } 51\end{array}$ & $\mathrm{H}$ & Cosp. \\
\hline \multicolumn{3}{|l|}{ Cannabaceae } \\
\hline Trema micrantha (L.) Blume INE & Á & NA, Méx, CA, SA. \\
\hline
\end{tabular}


Apéndice 1. Continuación

Familia/Especie/ Colector

Caricaceae

Carica papaya L. INE

Caryophyllaceae

Drymaria villosa Schltdl. \& Cham. Díaz-Gallegos 8

Celastraceae

Rhacoma macrocarpa (Brandegee) Standl. Matuda 3689

Zinowiewia rubra Lundell. Díaz-Gallegos 13

Chrysobalanaceae

Licania platypus (Hemsl.) Fritsch. Díaz-Gallegos 71

Clusiaceae

Calophyllum brasiliense Cambess. JMM 1920, Díaz-Gallegos 149

Clusia flava Jacq. Díaz-Gallegos 16

Clusia lundellii Standl. RMC, NMM y JMM s/n; JMM 1934

Combretaceae

Combretum fruticosum (Loefl.) Stuntz FVA $s / n$

Terminalia amazonia (J.F. Gmel.) Exell. HGD s/n; Díaz-Gallegos 14

Terminalia catappa L. HGD s/n

Connaraceae

Connarus lambertii (DC.) Sagot. Díaz-Gallegos 132

Convolvulaceae

Evolvulus alsinoides (L.) L. Díaz-Gallegos 132

Ipomoea alba L. INE

Ipomoea orizabensis (G. Pelletan) Ledeb. ex Steud, INE

Ipomoea squamosa Choisy. Matuda 3733

Merremia aturensis (Kunth) Hallier f.. Matuda 3739

Merremia tuberosa (L.) Rendle INE

Merremia umbellata (L.) Hallier f. INE

\section{Cucurbitaceae}

Momordica charantia L. INE

Psiguria triphylla (Miq.) C. Jeffrey GD et al. 20329; SZR s/n

Dilleniaceae

Curatella americana L. Pennington y Sarukhán s/n

Davilla nitida (Vahl) Kubitzki OFC 9

Ebenaceae

Diospyros digyna Jacq. Pennington y Sarukhán s/n

Erythroxylaceae

Erythroxylum guatemalense Lundell. Matuda 3706

Euphorbiaceae

Acalypha ferdinandii K. Hoffm. RFT y EL 40535; EFC et al., 2669, 2672; WSH 149, 153

Acalypha diversifolia Jacq. RMC y NMM 1125; EFC, EMS y

HC 1947; Díaz-Gallegos 181

Alchornea latifolia Sw. Pennington y Sarukhán s/n

Alchornea triplinervia (Spreng.) Müll. Arg. RMC, NMM Y JMM s/n

Cnidoscolus aconitifolius (Mill.) I. M. Johnst. INE

Croton bilibergianus Müll. Arg INE

Croton draco Schltdl. \& Cham. Pennington y Sarukhán s/n

Croton repens Schltdl. Matuda 3747

Euphorbia cyathophora Murray INE

Euphorbia thymifolia L. INE

FC Distribución

$\operatorname{Ar}$

$\mathrm{H}$

Á

Á

Á

Á

Á

Á

Á

Á

Á

Ar

$\mathrm{H}$

B

B

B

$\mathrm{H}$

B

B

B

B

Á

$\mathrm{H}$

Á

Á

Á

Á

Á

Á

Ar

Á

Á

Ar

$\mathrm{H}$

$\mathrm{H}$
NA, Méx, CA, SA, Car.

Cosp.

Méx.

Méx, Gua.

Méx, CA, SA.

Méx, CA, SA, Car.

Méx, CA.

Méx, CA, SA, Car.

Méx, CA, SA, Car.

Méx, CA, SA, Car.

Cosp.

Méx, CA, SA, Car.

Cosp.

Cosp.

Méx.

Méx, CA, SA.

Méx, CA, SA.

Méx, CA, SA.

Cosp.

Cosp.

Méx, CA, SA.

Méx, CA, SA, Car.

Méx, CA, SA, Car.

Méx, CA, SA.

Méx, CA.

Méx, CA, SA.

Méx, CA, SA.

Méx, CA, SA, Car.

Méx, CA, SA.

Méx, CA, SA.

Méx, CA.

Méx, CA.

Méx, CA.

Cosp.

Cosp. 
Apéndice 1. Continuación

\begin{tabular}{|c|c|c|}
\hline Familia/Especie/ Colector & FC & Distribución \\
\hline Garcia parviflora Lundell Díaz-Gallegos 180; LW 380 & Á & Méx. \\
\hline Hura polyandra Baill. Pennington y Sarukhán s/n & Á & Méx, CA. \\
\hline Manihot esculenta Crantz INE & $\mathrm{H}$ & Cosp. \\
\hline Sebastiania adenophora Pax \& K. Hoffm. Díaz-Gallegos 59 & Á & Méx, CA. \\
\hline Tragia mexicana Müll. Arg. Pennington y Sarukhán s/n & $\mathrm{B}$ & Méx, CA. \\
\hline \multicolumn{3}{|l|}{ Fabaceae } \\
\hline Acacia angustissima (Mill.) Kuntze JMM s/n; Díaz-Gallegos 172 & Á & NA, Méx, CA, SA. \\
\hline Acacia cornigera (L.) Willd. Díaz-Gallegos 178 & Á & NA, Méx, CA, SA. \\
\hline Acacia farnesiana (L.) Willd. Pennington y Sarukhán s/n & Á & Cosp. \\
\hline Acacia glomerosa Benth. HGD s/n & $\mathrm{Ar}$ & Méx, CA, SA. \\
\hline Acosmium panamense (Benth.) Yakovlev, HGD s/n & Á & Méx, CA, SA. \\
\hline Albizia leucocalyx (Britton \& Rose) L. Rico RMC, NMM Y JMM s/n & Á & Méx. \\
\hline Andira galeottiana Standl. EFC y HC 7028 & Á & Méx. \\
\hline Bauhinia ramosissima Benth. ex Hemsl. RMM y NMM 1197; Matuda 3676 & $\operatorname{Ar}$ & Méx. \\
\hline Cojoba arborea (L.) Britton \& Rose HGD s/n & Á & Méx, CA, SA. \\
\hline $\begin{array}{l}\text { Chamaecrista kunthiana (Schltdl. \& Cham.) H. S. Irwin \& Barneby. } \\
\text { Matuda } 3740,3751\end{array}$ & $\mathrm{H}$ & Méx, CA, SA. \\
\hline Dalbergia glabra (Mill.) Standl. TPR $s / n$ & Á & Méx, CA. \\
\hline Desmodium axillares (Sw.) DC. HGD s/n & $\mathrm{B}$ & Méx, CA, SA. \\
\hline Desmodium barbatum (L.) Benth. Matuda 3746 & $\mathrm{H}$ & Méx, CA, SA. \\
\hline Desmodium incanum (Sw.) DC. Díaz-Gallegos 282 & $\mathrm{H}$ & Cosp. \\
\hline Dialium guianense (Aubl.) Sandwith RMC, NMM y JMM s/n; Díaz-Gallegos 23 & Á & Méx, CA, SA, Car. \\
\hline Enterolobium cyclocarpum (Jacq.) Griseb. Pennington y Sarukhán s/n & Á & Méx, CA, SA, Car. \\
\hline Gliricidia sepium (Jacq.) Kunth ex. Walp. Díaz-Gallegos 372 & Á & Méx, CA, SA, Car. \\
\hline Inga oerstediana Benth. Ex Seem. HGD s/n & Á & Méx, CA, SA. \\
\hline Inga paterno Harms HGD 1883 & Á & Méx, CA. \\
\hline $\begin{array}{l}\text { Leucaena leucocephala (Lam.) de Wit RMC, NMM y JMM s/n, } \\
\text { Pennington y Sarukhán s/n }\end{array}$ & Á & Cosp. \\
\hline Lonchocarpus sericeus (Poir.) Kunth ex Dc. Pennington y Sarukhán s/n & Á & Méx, CA, SA. \\
\hline Lonchocarpus guatemalensis Benth INE, Díaz-Gallegos 279 & Á & Méx, CA. \\
\hline Lysiloma acapulcense (Kunth) Benth Díaz-Gallegos 281 & Á & Méx, CA. \\
\hline Lysiloma auritum (Schltdl.) Benth. Díaz-Gallegos 307 & Á & Méx, CA. \\
\hline Lysiloma latisiliquum (L.) Benth. Pennington y Sarukhán s/n & Á & Méx, CA, SA, Car. \\
\hline Macroptilium gracile (Poepp. Ex Benth.) Urb. Matuda 3761 & Á & Méx, CA, SA, Car. \\
\hline Mimosa somnians Humb. \& Bonpl. ex Willd Matuda 3734 & $\operatorname{Ar}$ & Méx, CA, SA. \\
\hline Myroxylon balsamum (L.) Harms. Pennington y Sarukhán s/n & Á & Méx, CA, SA. \\
\hline Stizolobium pruriens (L.) Medik INE & $\mathrm{B}$ & NA, Méx, CA, SA. \\
\hline Platymiscium yucatanum Standl. Pennington y Sarukhán s/n & Á & Méx. \\
\hline Piscidia piscipula (L.) Sarg. Pennington y Sarukhán s/n & Á & Méx. \\
\hline Pithecellobium donnell-smithii (Britton \& Rose) Standl. Matuda 3693 & Á & Méx, CA. \\
\hline Pterocarpus officinalis Jacq. HGD s/n & Á & Méx, CA, Car. \\
\hline Pterocarpus rohrii Vahl. Díaz-Gallegos 92 & Á & Méx, CA, SA, Car. \\
\hline Samanea saman (Jacq.) Merr. JMM s/n & Á & Cosp. \\
\hline Senna multijuga subsp. doylei (Britton \& Rose) Irwin \& Barneby EFC y HC 5275 & Á & Méx. \\
\hline Schizolobium parahyba (Vell.) S. F. Blake Díaz-Gallegos 215, HGD 1855 & Á & Méx, CA, SA. \\
\hline Swartzia guatemalensis (Donn. Sm.) Pittier INE & Á & Méx, CA. \\
\hline Tephrosia nitens Benth. Ex Seem. Matuda 3758 & $\mathrm{H}$ & Méx, CA, SA. \\
\hline Teramnus labialis (L.f.) Spreng. EFC et al. 1932 & $\mathrm{H}$ & Cosp. \\
\hline Vatairea lundellii (Standl.) Killip ex Recor Díaz-Gallegos 202, 115 & Á & Méx, CA. \\
\hline Zygia englesingii (Standl. Ex Record) Record. Matuda 3665 & Á & Méx, CA. \\
\hline
\end{tabular}


Apéndice 1. Continuación

Familia/Especie/ Colector

\section{Gentianaceae}

Coutoubea spicata Aubl. Matuda 3171

\section{Gesneriaceae}

Achimenes grandiflora (Schltdl.) DC. INE

Columnea schiedeana Schltdl. INE

Columnea sulfurea Donn. Sm. Matuda 3714

\section{Hippocrateaceae}

Salacia petenensis Lundell RMC, NMM y JMM 1198;JMM 1944

\section{Lamiaceae}

Scutellaria orichalcea Donn. Sm. Matuda 3681

Vitex gaumeri Greenm. Pennington y Sarukhán s/n

Loganiaceae

Spigelia humboldtiana Cham.\& Schltdl. RFT y EL 40523

Spigelia anthelmia L. INE

\section{Loranthaceae}

Struthanthus orbicularis (Kunth) Blume RMC, NMM y JMM 1216

Psittacanthus calyculatus (DC.) G. Don INE

\section{Lythraceae}

Cuphea carthagenensis (Jacq.) J. F. Macbr. Matuda 3749

\section{Malphigiaceae}

Bunchosia guatemalensis Nied. RMC, NMM y JMM 1217

Bunchosia lindeniana A. Juss. Díaz-Gallegos 230, 62

Byrsonima crassifolia (L.) Kunth Pennington y Sarukhán s/n

Malpighia glabra L. Díaz-Gallegos 153-264

\section{Malvaceae}

Apeiba tibourbou Aubl. Pennington y Sarukhán s/n

Bernoullia flammea Oliv. INE; Pennington y Sarukhán s/n

Pseudobombax ellipticum (Kunth) Dugand Díaz-Gallegos 2

Byttneria aculeata (Jacq.) Jacq. INE

Ceiba pentandra (L.) Gaertn. INE; Díaz-Gallegos 396

Corchorus hirtus L. RFT y EL 40574

Corchorus siliquosus L. OFC 1

Hampea trilobata Standl. Díaz-Gallegos 79, 98

Heliocarpus appendiculatus Turcz INE

Heliocarpus donnellsmithii Rose. Díaz-Gallegos 328

Helicteres guazumifolia Kunth OFC 4

Hibiscus costatus A. Rich. OFC 10; Matuda 3753

Luehea speciosa Willd. Pennington y Sarukhán $s / n$

Ochroma pyramidale (Cav. ex Lam.) Urb. HGD 1868; DEB s/n;

Pennington y Sarukhán s/n

Pachira aquatica Aubl. JMM s/n; DEB s/n

Pavonia schiedeana Steud. RFT \& EL 40568; Spellman et al., 202

Pavonia rosea Wall. ex Moris. JMM 1947; MJ y EFC 2415

Quararibea funebris (La Llave) Vischer EFC 5278; EFC y HC 6236; RFT y

EL 40546; Díaz-Gallegos 175, 09, 39

Trichospermum mexicanum (DC.) Baill. RFT s/n; EL 40571;

JMM 1907; Díaz-Gallegos 358

Sida acuta Burn OFC 15, Díaz-Gallegos 292.

Sterculia apetala (Jacq.) H. Karst. Pennington y Sarukhán s/n

FC

Distribución

$\mathrm{H}$

$\mathrm{H}$

$\mathrm{H}$

$\mathrm{H}$

Á

$\mathrm{H}$

Á

$\mathrm{H}$

$\mathrm{H}$

$\mathrm{H}$

$\mathrm{H}$

$\mathrm{H}$

Á

Á

Á

$\mathrm{Ar}$

Á

Á

Á

$\mathrm{H}$

Á

$\mathrm{H}$

$\mathrm{Ar}$

Á

Á

Á

Ar

$\mathrm{H}$

Á

Á

Á

$\mathrm{H}$

$\mathrm{H}$

Á

Á

$\mathrm{H}$

Á
Méx, CA, SA, Car.

Méx, CA.

Méx.

Méx, CA.

Méx, CA.

Méx, CA.

Méx, CA.

Méx, CA, SA.

Méx, CA, SA, Car.

Méx, CA, SA.

Méx, CA.

Méx, CA, SA.

Méx, CA.

Méx, CA, SA.

Méx, CA, SA.

Méx, CA, Car.

Méx, CA, SA.

Méx, CA.

NA, Méx, CA, SA, Car.

Méx, CA, SA.

Cosp.

NA, Méx, CA, SA, Car. NA, Méx, CA, SA, Car.

Méx, CA.

Méx, CA.

Méx, CA, Car.

Méx, CA.

Méx, CA, Car.

Méx, CA, SA.

Méx, CA, SA.

Cosp.

Méx, CA, SA, Car.

Méx, CA, Car.

Méx, CA.

Méx, CA, SA.

Cosp.

Méx, CA, SA. 
Apéndice 1. Continuación

Familia/Especie/ Colector

\section{Marcgraviaceae}

Souroubea loczyi (V.A. Richt.) de Roon. Matuda 3679

Melastomataceae

Bellucia grossularioides (L.) Triana. Matuda 3712

Clidemia capitellata (Bonpl.) D. Don Matuda 3672

Clidemia dependens Pav. Ex D. Don Matuda 3663

Clidemia octona (Bonpl.) L.O. Williams INE

Miconia affinis DC. Pennington y Sarukhán s/n

Miconia argentea (Sw.) DC. RMC, NMM y JMM s/n; Pennington y Sarukhán s/n

Miconia ciliata (Rich.) DC. Díaz-Gallegos 397

Miconia ibaguensis (Bonpl.) Triana. Matuda 3687

Miconia mexicana (Bonpl.) Naudin INE

Miconia tomentosa (Rich.) D. Don ex DC. INE

Miconia triplinervis Ruíz \& Pav. Díaz-Gallegos 157, 142

Meliaceae

Cedrela odorata L. RFT y EL 40547, Díaz-Gallegos 312

Guarea glabra Vahl JMM s/n; Díaz-Gallegos 136, 184, 210, 97, 146

Guarea grandifolia C. Dc. Díaz-Gallegos 129

Swietenia macrophylla King Díaz-Gallegos 156

Trichilia havanensis Jacq. RMC, NMM y JMM 1206; Pennington y Sarukhán s/n

Trichilia breviflora S. F. Blake \& Standl. INE

Trichilia martiana C. DC. INE, EFC 291, EFC y HC s/n

Trichilia pallida Sw. Díaz-Gallegos 132, 45, 166, 46, 99; EFC y HC 6238, 5291

Menispermaceae

Cissampelos pareira L. INE

Cissampelos tropaeolifolia DC. Matuda 3686

Moraceae

Artocarpus altilis (Parkinson) Fosberg. HGD 1866; Díaz-Gallegos 308

Brosimum alicastrum Sw. HGD 1874; Díaz-Gallegos 34

Castilla elastica Sessé INE; Díaz-Gallegos 196

Clarisia biflora Ruiz \& Pav. RFT y EL 40536, 40540

Coussapoa oligocephala Donn. Sm. RMC, NMM y JMM s/n

Dorstenia contrajerva L. OFC 16; RFT s/n; EL 40524, 6546;

Díaz-Gallegos 222; EFC, EM y HC 1928; GD et al. 20349

Dorstenia drakena L. JMM 1913

Ficus aurea Nutt. JMM 1905

Ficus citrifolia Mill. Díaz-Gallegos 217

Ficus crassinervia Desf. Ex Willd. RFT y EL 40533

Ficus cotinifolia Kunth INE

Ficus insipida Willd. RFT y EL40548; López-Mendoza s/n;

JMM 1903; GD et al. 20355

Ficus maxima Mill. Díaz-Galllegos 301

Ficus pertusa L. F. HGD 1876

Ficus yoponensis Desv. EFC s/n.

Poulsenia armata (Miq.) Standl. JMM 1930; JMM 1948; HGD s/n;

Díaz-Gallegos 37; GD et al. 20356

Pseudolmedia glabrata (Liebm.) C. C. Berg. Díaz-Gallegos 57, 38, 258, 78

Trophis racemosa (L.) Urb. INE; Díaz-Gallegos 74, 226, 222, 268.

\section{Moringaceae}

Moringa oleifera Lam. Díaz-Gallegos 224

FC Distribución

B

Á

$\mathrm{H}$

Ar

Ar

Ar

Á

Ar

Ar

Ar

Á

Ar

Á

Á

Á

Á

Á

Á

Á

Á

B

B

Á

Á

Á

Á

Á

$\mathrm{H}$

$\mathrm{H}$

Á

Á

Á

Á

Á

Á

Á

Á

Á

Á

Á

Á
Méx, CA.

Méx, CA, SA, Car.

Méx, CA, SA, Car.

Méx, CA, SA, Car.

Méx, CA, SA.

NA, Méx, CA, SA, Car.

Méx, CA.

Méx, CA, SA.

Méx, CA, SA.

Méx, CA.

Méx, CA, SA, Car.

Méx, CA, SA.

Méx, CA, SA, Car.

Méx, CA, SA.

Méx, CA, SA.

Méx, CA, SA.

Méx, CA, SA.

Méx, CA.

Méx, CA, SA, Car.

Méx, CA, SA, Car.

Cosp.

Méx, CA, SA.

Cosp.

NA, Méx, CA, SA, Car.

Cosp.

Méx, CA, SA.

Méx, CA.

Méx, CA, SA, Car.

Méx, CA.

NA, Méx, CA.

NA, Méx, CA, SA, Car.

Méx, CA, Car.

Méx, CA.

Méx, CA, SA.

Méx, CA, SA, Car.

Méx, CA, SA.

Méx, CA, SA.

Méx, CA, SA.

Méx, CA.

Méx, CA, SA, Car.

Cosp. 
Apéndice 1. Continuación

Familia/Especie/ Colector

Myrtaceae

Eugenia aeruginia D. C. Díaz-Gallegos 137

Eugenia breedlovei Barrie RMC, NMM y JMM s/n

Eugenia capuli (Schltdl. \& Cham.) Hook. \& Arn. Pennington y Sarukhán s/n

Eugenia mexicana Steud. Pennington y Sarukhán s/n

Eugenia oerstediana O. Berg. INE

Eugenia origanoides O. Berg. JMM 1921

Myrcia splendens (Sw.) DC. Díaz-Gallegos 42

Pimenta dioica (L.) Merr. Pennington y Sarukhán s/n

Psidium guajava L. Pennington y Sarukhán s/n

\section{Nyctaginaceae}

Neea psychotrioides Donn. Sm. JMM 1945; Díaz-Gallegos 189

Neea amplifolia Donn. Sm. RMC, NMM y JMM 1999

Pisonia aculeata L. Díaz-Gallegos 263

\section{Olacaceae}

Heisteria media S. F. Blake Matuda 3690

\section{Oxalidaceae}

Biophytum dendroides (Kunth) DC. Wendt $s / n$

Passifloraceae

Passiflora hahnii (E. Fuern.) Mast DD 357

Phyllanthaceae

Phyllanthus amarus Schumach. \& Thonn. INE

\section{Polygonaceae}

Coccoloba barbadensis Jacq. Pennington y Sarukhán s/n

Coccoloba belizensis Standl. Díaz-Gallegos 333

Coccoloba escuintlensis Lundell. Díaz-Gallegos 148

Coccoloba tuerckheimii Donn. Sm. INE

Polygonum lapathifolium L. INE

Polygonum punctatum Elliot INE

Polygala longicaulis Kunth OFC 11

Primulaceae

Ardisia pellucida Oerst OFC 23

Ardisia paschalis Donn. Sm. RMC, NMM y JMM 1202; Díaz-Gallegos 224

Ardisia verapazensis Donn. Sm. Díaz-Gallegos 53

Parathesis leptopa Lundell JMM 1912

Rapanea guianensis Aubl. Díaz-Gallegos 241

\section{Rubiaceae}

Alibertia edulis (Rich.) A. Rich. Ex Dc. Díaz-Gallegos 141, 348

Alseis yucatanensis Standl. Díaz-Gallegos 164

Augusta rivalis (Benth.) J. H. Kirkbr. Matuda 3673

Blepharidium guatemalense Standl. Matuda 3670

Borreria laevis (Lam.) Griseb INE

Calycophyllum candidissimum (Vahl.) DC. Pennington y Sarukhán s/n

Coccocypselum hirsutum Bartl. ex DC. Matuda 3667

Chione chiapasensis Standl. Matuda 3906, 3675

Chomelia protracta (Bartl. ex DC.) Standl. Matuda 3730

Faramea occidentalis (L.) A. Rich. RMC y NMM 1204; Díaz-Gallegos 227,235, 127

Genipa americana L. INE

Geophila repens (L.) I.M. Johnst. JMM s/n

Guettarda combsii Urb. Matuda 3703
FC

Distribución

Méx, CA, Car.

Chis.

Méx, CA.

Méx, CA.

Méx, CA, SA.

Méx, CA, SA.

Méx, CA, SA.

Méx, CA, Car.

NA, Méx, CA, SA, Car.

Méx, CA.

Méx, CA.

NA, Méx, CA, SA, Car.

Méx, CA.

Méx, CA, SA.

Méx, CA, SA.

Cosp.

Méx, CA.

Méx, CA.

Méx, CA.

Méx, CA.

Cosp.

Méx, CA, SA.

Méx, CA, SA, Car.

Méx, CA, SA.

Méx, CA, SA.

Méx, Gua.

Méx, CA, SA.

NA, Méx, CA, SA.

Méx, CA, SA.

Méx, CA.

Méx, CA.

Méx, CA.

NA, Méx, CA, SA, Car.

Méx, CA, SA, Car.

Méx, CA, SA.

Chis.

Méx, CA.

Méx, CA, SA, Car.

Méx, CA, SA.

Méx, CA, SA, Car.

Méx, CA, Car. 
Apéndice 1. Continuación

\section{Familia/Especie/ Colector}

Hamelia axillaris Sw. EFC y HC 7011, 5284, 6235; EFC, OC y HC 5614

Hamelia barbata Standl. Díaz-Gallegos 105, 101

Hamelia patens Jacq. INE

Posoqueria latifolia (Rugne) Schult. Matuda 3707

Psychotria brachiata Sw. Matuda 3664

Psychotria chiapensis Standl. JMM 1942; Matuda 3711; WSH 138

Psychotria acuminata Benth. JMM 1923; Matuda 3608

Palicourea domingensis (Jacq.) DC. RMC, NMM y JMM 1995; JMM 1919

Psychotria flava Oerst. ex Standl. Matuda 3711

Psychotria limonensis K. Krause RFT y EL 40561;

Díaz-Gallegos 14. 90, 112; WSH 130

Psychotria officinalis (Aubl.) Raeusch. Ex Sandwith Matuda 3623

Psychotria pubescens Sw. RMC, NMM y JMM s/n; EFC 1924;

EFC y HC s/n; WSH 131

Psychotria simiarum Standl. EFC, HC y OC s/n

Psychotria trichotoma M. Martens \& Galeotti. Díaz-Gallegos 207

Randia aculeata L. INE

Richardia scabra L. Matuda 3659

Simira salvadorensis (Standl.) Steyerm. Pennington y Sarukhán s/n

\section{Rutaceae}

Esenbeckia pentaphylla (Macfad.) Griseb. Díaz-Gallegos 147

Citrus maxima (Burm.) Merr. INE

Zanthoxylum panamense P. Wilson Matuda 3708

Zanthoxylum riedelianum Engl. Pennignton y Sarukhán s/n

\section{Salicaceae}

Casearia commersoniana Cambess. Matuda 3715

Casearia nitida (L.) Jacq. DD355, RMC, NMM y JMM 1209; Díaz-Gallegos 152

Laetia thamnia L. Matuda 3715

Pleuranthodendron lindenii (Turcz.) Sleumer EMS s/n; EFC, HC y OC s/n

Zuelania guidonia (Sw.) Britton \& Millsp. Díaz-Gallegos 81

Santalaceae

Phoradendron carneum Urb. INE

Phoradendron pedicellatum (Tiegh.) Kuijt Matuda 3727

Phoradendron piperoides (Kunth) Trel. Matuda 3721

\section{Sapindaceae}

Cupania dentata DC. Díaz-Gallegos 269

Cupania glabra Sw. INE

Cupania silvatica Casar. Díaz-Gallegos 251

Sapindus saponaria L. Díaz-Gallegos 131

\section{Sapotaceae}

Manilkara zapota (L.) P. Royen. INE

Chrysophyllum cainito L. HGD 1880

Chrysophyllum mexicanum Brandegee ex Standl. Pennington y Sarukhán s/n

Pouteria campechiana (Kunth) Baehni Díaz-Gallegos 213, 56

Pouteria reticulata (Engl.) Eyma Pennington y Sarukhán s/n

Pouteria sapota (Jacq.) H. E. Moore \& Stearn Pennington y Sarukhán s/n

Sideroxylon persimile (Hemsl.) T.D. Penn Pennington y Sarukhán s/n

\section{Simaroubaceae}

Simarouba glauca DC. Díaz-Gallegos 202

\section{FC}

$\mathrm{H}$

$\mathrm{Ar}$

$\mathrm{H}$

Á

$\operatorname{Ar}$

$\operatorname{Ar}$

$\operatorname{Ar}$

$\operatorname{Ar}$

Á

$\operatorname{Ar}$

Á

Á

Á

Á

Ar

$\mathrm{H}$

Á

Á

Á

Á

Á

Á

Ar

Á

Á

Á

$\mathrm{H}$

$\mathrm{H}$

$\mathrm{H}$

Á

Á

Á

Á

Á

Á

Á

Á

Á

Á

Á

Á
Distribución

Méx, CA, SA, Car.

Chis, Gua.

Cosp.

Méx, CA, SA, Car.

Méx, CA, SA, Car.

Méx, CA.

Méx, CA, SA, Car.

Méx, CA, SA, Car.

Méx, CA.

Méx, CA.

Méx, CA, SA.

Méx, CA, SA.

Méx, CA.

Méx, CA, SA.

Méx, CA, SA, Car.

NA, Méx, CA, SA.

Méx, CA.

Méx, CA.

Méx, CA, SA.

Méx, CA.

Méx, CA, SA.

Méx, CA, SA.

Méx, CA, SA.

Méx, CA, Car.

Méx, CA.

Méx, CA.

Méx.

Méx, CA.

Méx, CA, SA, Car.

Méx, CA.

NA, Méx, CA, SA.

NA, Méx, CA, SA.

Cosp.

NA, Méx, CA, SA, Car.

NA, Méx, CA, SA, Car.

Méx, CA.

NA, Méx, CA, SA, Car.

NA, Méx, CA, SA, Car.

Méx, CA, Car.

Méx, CA, SA, Car.

NA, Méx, CA, Car. 
Apéndice 1. Continuación

\section{Familia/Especie/ Colector}

\section{Solanaceae}

Capsicum annuum L. EFC y HC s/n

Cestrum guatemalense Francey. HGD s/n

Cestrum nocturnum L. EFC y HC s/n

Jaltomata procumbens (Cav.) J.L. Gentry INE

Juanulloa mexicana (Schltdl.) Miers RFT y EL 40545

Lycianthes amatitlanensis (J.M. Coult. \& Donn. Sm.) Bitter JMM 1943

Lycianthes heteroclita (Sendtn.) Bitter GD et al. 20333; EFC, OC

y HC 5604; RMC y NMM 1192

Lycianthes purpusii (Brandegee) Bitter. WHS 126; GD et al. 20331

Solanum americanum Jacq. INE

Solanum rovirosanum Donn. Sm. EFC y HC 5297

Solanum torvum Sw. INE

\section{Surianaceae}

Recchia simplicifolia T. Wendt \& E. J. Lott INE

Urticaceae

Cecropia peltata L. OFC s/n

Cecropia obtusifolia Bertol INE; Díaz-Gallegos 374

Myriocarpa longipes Liebm. HGD 1872; WSH 140

Myriocarpa obovata Donn. Sm, INE

Urera caracasana (Jacq.) Gaudich. Ex Griseb. Díaz-Gallegos 75-44, 358

Urera baccifera (L.) Gaudich. ex Wedd. JMM 1941; RMC, NMM y JMM s/n

Urera elata (Sw.) Griseb. OFC 18, 19; EFC, EM y HC 1950; EFC, OC y

HC 5611; EFC y HC 7001; Matuda 3699

\section{Verbenaceae}

Lippia dulcis Trevir. JMM 1916

Priva lappulacea (L.) Pers. INE

Stachytarpheta angustifolia (Mill.) Vahl OFC 6

Violaceae

Rinorea hummelii Sprague OFC 12; Díaz-Gallegos 44; MJH y EFC 2407; MJH 141

Rinorea guatemalensis (S. Watson) Bartlett SZR s/n, HGD 1879; RMC y

NMM 1201; Díaz-Gallegos 29

\section{Vitaceae}

Ampelocissus erdvenbergiana Planch. Matuda 3201

Vochysiaceae

Vochysia guatemalensis Donn. Sm. EFC y HC 7029

Ximeniaceae

Ximenia americana L. Pennington y Sarukhán s/n

\section{MAGNOLIOPSIDA}

\section{Annonaceae}

Annona cherimolla Mill INE, Pennington y Sarukhán s/n

Annona muricata L. Díaz-Gallegos 159

Annnona reticulata L. Pennington y Sarukhán $s / n$

Cymbopetalum baillonii R.E. Fr. DEB y FSK s/n; RMC y NMM 1188; DD 355a

Cymbopetalum penduliflorum (Dunal) Baill. JMM 1925, Pennington y Sarukhán s/n

Guatteria amplifolia Triana \& Planch. RMC y NMM 1212, Matuda 3684, 3685

Guatteria anomala R.E. Fr. JMM 1925, Matuda, Díaz-Gallegos 03

Malmea depressa (Baill.) R. E. Fr. INE
FC

Distribución

Cosp.

Méx, Gua.

Cosp.

NA, Méx, CA.

Méx, CA.

Méx, CA, SA.

Méx, CA.

Méx, CA.

Cosp.

Méx, CA, SA.

Cosp.

Méx, CA.

Méx, CA, SA, Car.

Méx, CA.

Méx, CA.

Méx, CA.

Méx, CA.

Méx, CA, SA.

Méx, CA, SA.

Méx, CA, Car. NA, Méx, CA, Car. Méx, CA, SA, Car.

Méx, CA.

Méx, CA.

Méx, CA.

Méx, CA, SA.

Cosp.

Cosp.

Cosp.

Cosp.

Méx.

Méx, CA.

Méx, CA.

Méx, CA.

Méx, CA. 
Apéndice 1. Continuación

\begin{tabular}{|c|c|c|}
\hline Familia/Especie/ Colector & FC & Distribución \\
\hline Rollinia mucosa (Jacq.) Baill. JMM 1929; Díaz-Gallegos 159 & Á & Méx, CA. \\
\hline Rollinia rensoniana Standl. INE & Á & Méx, CA. \\
\hline \multicolumn{3}{|l|}{ Aristolochiaceae } \\
\hline Aristolochia odoratissima L. López-Mendoza s/n & $\mathrm{B}$ & Méx, CA, SA, Car. \\
\hline \multicolumn{3}{|l|}{ Lauraceae } \\
\hline Beilschmiedia anay (S. F. Blake) Kosterm. Pennington y Sarukhán s/n & Á & Méx, CA. \\
\hline Licaria peckii (I. M. Johnst.) Kosterm. Díaz-Gallegos 22 & Á & Méx, CA. \\
\hline Nectandra ambigens (S.F. Blake) C. K. Allen. Pennington y Sarukhán s/n & Á & Méx, CA. \\
\hline Nectandra cissiflora Nees. Díaz-Gallegos 185, 124 & Á & Méx, CA, SA. \\
\hline Nectandra leucocome Rohwer RMC, NMM y JMM s/n & Á & Chis. \\
\hline Nectandra nitida Me. Díaz-Gallegos 154 & Á & Méx, CA, SA. \\
\hline Nectandra reticulata (Ruiz \& Pav.) Mez, JMM 1927 & Á & Méx, CA, SA. \\
\hline Ocotea magnifolia (Lundell) Lundell RFT y EL 40526; EFC y HC 3118 & Á & Méx, CA. \\
\hline Persea americana Miller. Díaz-Gallegos 21 & Á & Méx, CA, SA. \\
\hline \multicolumn{3}{|l|}{ Monimiaceae } \\
\hline Mollinedia pallida Lundell JMM s/n & Á & Méx, Gua. \\
\hline Mollinedia viridiflora Tul. Díaz-Gallegos 245, 114 & Á & Méx, CA. \\
\hline \multicolumn{3}{|l|}{ Piperaceae } \\
\hline Peperomia macrostachyos (Vahl) A. Dietr. RFT y EL 4055; EFC y HC; WSH 163 & $\mathrm{H}$ & Méx, CA, SA. \\
\hline Peperomia deppeana Schltdl. \& Cham. GD et al. 20341 & $\mathrm{H}$ & Méx, CA. \\
\hline Peperomia glabella (Sw.) A. Dietr. RMC, NMM y JMM 1215 & $\mathrm{H}$ & Méx, CA, SA. \\
\hline Peperomia urocarpa Fisch. \& C. A. Mey. Matuda 3214, 3671; WSH 154 & $\mathrm{H}$ & Méx, CA, SA. \\
\hline Piper aequale Vahl. Díaz-Gallegos 327 & $\mathrm{Ar}$ & Méx, CA, SA. \\
\hline Piper amalago L. JMM 1917; Díaz-Gallegos 35, 108, 80, 228 & $\mathrm{Ar}$ & Méx, CA, SA, Car. \\
\hline Piper auritum Kunth Díaz-Gallegos 327 & Ar & NA, Méx, CA. \\
\hline Piper curvatipes Trel. RMC y NMM 1193 & $\operatorname{Ar}$ & Méx, Gua. \\
\hline Piper flavidum C. DC. RMC, NMM y JMM 1210 & $\mathrm{Ar}$ & Méx, Gua. \\
\hline Piper hispidum Sw. JMM 1909 & $\operatorname{Ar}$ & Méx, CA, SA. \\
\hline Piper matudae Lundell Matuda 3697 & $\mathrm{Ar}$ & Chis. \\
\hline Piper peltatum L. RMC y NMM 1205; WSH 159 & $\mathrm{Ar}$ & Méx, CA, SA. \\
\hline \multicolumn{3}{|l|}{ Siparunaceae } \\
\hline Siparuna andina (Tul.) A. DC. Díaz-Gallegos 160, 121 & Á & Méx, CA, SA. \\
\hline
\end{tabular}

\section{MONOCOTILEDÓNEAS}

\section{Araceae}

Anthurium flexile Schott, RMC y NMM s/n

Anthurium pentaphyllum var. bombacifolium (Schott) Madison, HGD 1925

Anthurium scandens (Aubl.) Engl. JMM 1224; RMC, NMM y JMM 1951, EFC 7019

Anthurium schlechtendalii Kunth RMC, NMM y JMM s/n

Dieffenbachia oerstedii Schott. JMM 1910

Monstera acuminata K. Koch RMC, NMM y JMM s/n

Monstera deliciosa Leibm. Díaz-Gallegos 14, 378

Monstera tuberculata Lundell RMC y $N M M s / n$

Philodendron aurantiifolium subsp. calderense (K. Krause) Gray JMM 1911

Philodendron radiatum Schott. INE

Philodendron sagittifolium Liebm INE

Spathiphyllum friedrichsthalii Schott INE

Spathiphyllum cochlearispathum (Liebm.) Engl., INE

Syngonium podophyllum Schott Díaz-Gallegos 373

Xanthosoma robustum Schott. RMC y NMM s/n; Díaz-Gallegos 333

$\begin{array}{ll}\text { Méx, CA. } \\ \text { Méx, CA. } \\ \text { Méx, CA. } \\ \text { Méx, CA. } \\ \text { Béx, CA. } \\ \text { Bosp. } \\ \text { B } \\ \text { Méx, CA. } \\ \text { B } \\ \text { Méx, CA. } \\ \text { H } & \text { Méx, CA. } \\ \text { B } & \text { Méx, CA. } \\ \text { H } & \text { Méx. } \\ & \text { NA, Méx, CA, SA. } \\ & \text { Méx, CA. }\end{array}$


Apéndice 1. Continuación

Familia/Especie/ Colector

Arecaceae

Astrocaryum mexicanum Liebm. Díaz-Gallegos 30, 123

Bactris major Jacq. RMC, NMM y JMM 1227

Cryosophila stauracantha (Heynh.) R. Evans. Díaz- Gallegos 144

Chamaedorea arenbergiana H. Wendl. RMC, NMM y JMM s/n

Chamaedorea cataractarum Mart. RMC, NMM y JMM s/n

Chamaedorea elegans Mart. RMC, NMM y JMM s/n, Díaz-Gallegos 176, 13

Chamaedorea ernesti-angustii $\mathrm{H}$. Wendl. DEB y RLD 29778, RMC y

NMM s/n; Díaz-Gallegos 67,65

Chamaedorea neurochlamys Burret TBC 40552, OFC 17

Chamaedorea oblongata Mart. RMC, NMM y JMM s/n

Chamaedorea tepejilote Liebm. ex Mart. GD et al., 20328; RMC, NMM y

JMM s/n, Díaz-Gallegos 63

Desmoncus orthacanthos Mart. Díaz-Gallegos 5, HGD s/n

Sabal muritiiformis (H. Karst.) Griseb. \& H. Wedl. Pennington y Sarukhán s/n

Bromeliaceae

Aechmea bracteata (Sw.) Griseb. RMC, NMM y JMM s/n

Aechmea mexicana Baker INE

Aechmea tillandsioides (Mart. Ex Schult. F) Baker. Díaz-Gallegos 340

Catopsis sessiliflora (Ruíz \& Pav.) Me. Díaz-Gallegos 299

Picairnia recurvata (Scheidw.) K. Koch. INE

Podaechmea lueddmanniana (K. Koch) L. B. Sm. \& W J. Kress INE

Tillandsia juncea (Ruiz \& Pav.) Poir. INE

Tillandsia caput-medusae E. Morren INE

Tillandsia polystachia (L.) L. INE

Tillandsia schiedeana Steud. JMM 1949; RMC, NMM y JMM s/n

Cannaceae

Canna indica L. Díaz- Gallegos 317

Commelinaceae

Callisia repens (Jacq.) L. SZR $s / n$

Commelina diffusa Burm. f. INE

Commelina erecta L. INE

Commelina tuberosa L. INE

Tradescantia zanonia (L.) Sw. RFT y EL 40566, 40532, SZR 169

Tradescantia zebrina Heynh. ex Bosse EFC, HC y EMS s/n

Tripogandra serrulata (Vahl) Handlos INE

Costaceae

Costus pulverulentus C. Presl. RFT y EL 40573

Cyperaceae

Cyperus odoratus L., Díaz-Gallegos 287

Cyperus unioloides (R. Br.) Urb., Matuda 3744

Scleria melaleuca Rchb. ex Schltdl \& Cham. OFC 3

Dioscoreaceae

Dioscorea bartlettii C. V, Morton, Matuda 3669

Dioscorea macrostachya Benth, Díaz-Gallegos 351

Haemodoraceae

Xiphidium caeruleum Aubl., Matuda 3701.

Heliconiaceae

Heliconia subulata Ruiz \& Pav. JMM s/n

Heliconia adflexa (Griggs) Standl. INE
FC

Distribución

Méx, CA.

Méx, CA.

Méx, CA.

Méx, CA.

Chis, Oax.

Méx, CA.

Méx, CA.

Méx, CA.

Méx, CA.

Méx, CA.

Méx, CA, SA, Car.

Méx, CA, SA.

Méx, CA, SA.

Méx, CA.

Méx, CA.

Méx, CA, SA, Car.

Méx, CA.

Méx, CA.

Méx, CA, SA, Car.

Méx, CA.

Méx, CA, SA, Car.

Méx, CA, SA, Car.

Cosp.

NA, Méx, CA, SA, Car.

Cosp.

Méx, CA, SA.

NA, Méx, CA, SA, Car.

NA, Méx, CA, SA, Car.

Méx, CA.

Méx, CA, SA.

Méx, CA, SA.

Cosp.

Cosp.

Cosp.

Méx, CA.

Méx, CA.

Méx, CA, SA.

Méx, CA, SA.

Méx, CA. 
Apéndice 1. Continuación

\begin{tabular}{|c|c|c|}
\hline Familia/Especie/ Colector & FC & Distribución \\
\hline $\begin{array}{l}\text { Heliconia aurantiaca Ghiesbr. RMC y JMM 1189; } \\
\text { Díaz-Gallegos 13, 332, WSH } 127\end{array}$ & $\mathrm{H}$ & Méx, CA. \\
\hline Heliconia bihai (L.) L. INE & $\mathrm{H}$ & Méx, CA, SA, Car. \\
\hline Heliconia librata Griggs, JMM 1902 & $\mathrm{H}$ & Méx, CA. \\
\hline Heliconia schiedeana Klotzsch, INE & $\mathrm{H}$ & Méx, Gua. \\
\hline Heliconia vaginalis Benth INE & $\mathrm{H}$ & Méx, CA. \\
\hline \multicolumn{3}{|l|}{ Iridaceae } \\
\hline Cipura paludosa Aubl. Matuda 3729 & $\mathrm{H}$ & Méx, CA, SA. \\
\hline \multicolumn{3}{|l|}{ Marantaceae } \\
\hline Calathea micans (L. Mathieu) Körn. RFT s/n; EL 40530 & $\mathrm{H}$ & Méx, CA, SA. \\
\hline Maranta arundinacea L. OFC 7 & $\mathrm{H}$ & Cosp. \\
\hline Pleiostachya pruinosa (Regel) K. Schum. Matuda 3680 & $\mathrm{H}$ & Méx, Gua. \\
\hline $\begin{array}{l}\text { Stromanthe macrochlamys (Woodson \& Standl.) H. Kenn. \& } \\
\text { Nicolson Díaz-Gallegos } 363\end{array}$ & $\mathrm{H}$ & Méx, Gua. \\
\hline \multicolumn{3}{|l|}{ Musaceae } \\
\hline Musa paradisiaca L. INE & $\mathrm{AB}$ & Cosp. \\
\hline \multicolumn{3}{|l|}{ Orchidaceae } \\
\hline Epidendrum ramosum Jacq. JMM 1904; RMC, NMM y JMM s/n & $\mathrm{H}$ & NA, Méx, CA, SA, Car. \\
\hline Epidendrum rigidum Jacq. JMM 1936; RMC, NMM y JMM s/n & $\mathrm{H}$ & NA, Méx, CA, SA, Car. \\
\hline Isochilus mayor Schltdl. \& Cham. Díaz-Gallegos 2 & $\mathrm{H}$ & Méx, CA, SA, Car. \\
\hline Nidema boothii (Lindl.) Schltr. JMM 1937; RMC, NMM y JMM s/n & $\mathrm{H}$ & Méx, CA. \\
\hline Notylia barkeri Lindl. INE & $\mathrm{H}$ & Méx, Gua. \\
\hline Oncidium carthagenense (Jacq.) Sw. RMC, NMM y JMM s/n & $\mathrm{H}$ & NA, Méx, CA, SA, Car. \\
\hline Prosthechea cochleata (L.) Dressler INE & $\mathrm{H}$ & Méx, Gua. \\
\hline Specklinia grobyi (Bateman ex Lindl.) F. Barros INE & $\mathrm{H}$ & Méx, CA, SA, Car. \\
\hline \multicolumn{3}{|l|}{ Poaceae } \\
\hline Andropogon bicornis L. Matuda 3726, Díaz-Gallegos 302 & $\mathrm{H}$ & NA, Méx, CA, SA, Car. \\
\hline Andropogon selloanus (Hack.) Hack. Matuda 3741 & $\mathrm{H}$ & Méx, CA, SA, Car. \\
\hline Axonopus fissifolius (Raddi) Kuhlm Matuda 3737 & $\mathrm{H}$ & NA, Méx, CA, SA, Car. \\
\hline Eriochloa punctata (I.) Desv. Ex Ham. Matuda 3759 & $\mathrm{H}$ & Méx, CA, SA. \\
\hline Homolepis aturensis (Kunth) Chase, Matuda 3736 & $\mathrm{H}$ & Méx, CA, SA, Car. \\
\hline Lasiacis nigra Davidse INE & $\mathrm{H}$ & Méx, CA, SA. \\
\hline Lasiacis procerrima (Hack.) Hitchc. Matuda 3718 & $\mathrm{H}$ & Méx, CA, SA. \\
\hline Mesosetum blakei Swallen Matuda 3738 & $\mathrm{H}$ & Méx, CA. \\
\hline Oplismenus hirtellus (L.) P. Beauv. EFC y HC 1949; GD et al 20342 & $\mathrm{H}$ & Cosp. \\
\hline Panicum virgatum L. Matuda 3722 & $\mathrm{H}$ & Cosp. \\
\hline Panicum stoloniferum Poir. OFC 20; EFC 7002 & $\mathrm{H}$ & Méx, CA, SA. \\
\hline Panicum trichoides Sw OFC 21 & $\mathrm{H}$ & Cosp. \\
\hline Leptocoryphium lanatum (Kunth) Nees Matuda 3718 & $\mathrm{H}$ & NA, Méx, CA, SA, Car. \\
\hline Pentarrhaphis scabra Kunth Matuda 3742 & $\mathrm{H}$ & Méx, CA. \\
\hline Schizachyrium condensatum (Kunth) Nees Matuda 3741 & $\mathrm{H}$ & Méx, CA, SA, Car. \\
\hline Urochloa maxima (Jacq.)R. D. Webster INE & $\mathrm{H}$ & NA, Méx, CA, SA, Car. \\
\hline \multicolumn{3}{|l|}{ Pontederiaceae } \\
\hline Pontederia lanciolata Wall. Ex Kunth. Matuda 3688 & $\mathrm{H}$ & NA, Méx, CA, Car. \\
\hline \multicolumn{3}{|l|}{ Smilacaceae } \\
\hline Smilax lanceolata L. Matuda 3674 & $\mathrm{~B}$ & NA, Méx, CA, Car. \\
\hline Smilax spinosa Mill. Matuda 3709 & $\mathrm{~B}$ & Méx, CA, SA. \\
\hline
\end{tabular}


Apéndice 2. Relación de colectores de flora del Parque Nacional Palenque

Colector

David Dreyer

David L. Spellman, John D. Dwyer, Joseph E. Vaughan y Richard P. Wunderlin

Dennis E. Breedlove y Robert L. Dressler

E. Lathrop W.

Edgar Francisco Cabrera

Eizi Matuda

Esteban Manuel Martínez Salas

F. S. Kawahara

Francisco Ventura A.

George Eduard Caecilie

Gerrit Davidse, Mario Sousa S., Arthur O. Chater \& Edgar Fco. Cabrera C.

H. de Cabrera

Héctor Gómez Domínguez

Instituto Nacional de Ecología

Jorge Martínez Meléndez

José Reyes Díaz Gallegos

Michael J. Huft

Nayeli Martínez Meléndez

Oscar F. Clarke

R. López Mendoza

Robert Folger Thorme

Robert Folger Thorme

Robert L. Dressler

Rubén Martínez Camilo

Seler Caecilie

Sergio Zamudio Ruíz

Thennilapuram Parasuraman Ramammoorthy

Thomas B. Croat

W. Scott Hoover
Abreviatura

DD

Spellman et al

DEB y RLD

EL

EFC

Matuda

EMS

FSK

FVA

GEC

GD et al

$\mathrm{HC}$

HDG

INE

JMM

Díaz- Gallegos

$\mathrm{MJH}$

NMM

OFC

López-Mendoza

RFT

RFT

RLD

RFC

$\mathrm{SC}$

SZR

TPR

TBC

WSH 